\title{
A novel CXCL8 analog is effective in inhibiting the growth via cell cycle arrest and attenuating invasion of Lewis lung carcinoma
}

This article was published in the following Dove Press journal: OncoTargets and Therapy

\author{
Su-Ya Hsu' \\ Hui-Yuan $\mathrm{Yu}^{2}$ \\ Wei-Chen Lee ${ }^{\prime}$ \\ Chia-En Hsiao' \\ Chih-Lung Wu' \\ Hsi-Tsung Cheng ${ }^{2}$ \\ Li-Jin $\operatorname{Lin}^{2}$ \\ Fang $\mathrm{Li}^{3}$ \\ Yu-Ting Chou' \\ Jya-Wei Cheng'
}

'Department of Medical Science, Institute of Biotechnology, National Tsing Hua University, Hsinchu 300, Taiwan;

${ }^{2}$ Division of Cancer Research, Rise Biopharmaceuticals Inc., Zhongguancun Shangdi Bio-medical Park, Beijing I00085, People's Republic of China; ${ }^{3}$ Department of Immunology, Dalian Medical University, Dalian I 16044, People's Republic of China

Correspondence: Yu-Ting Chou; Jya-Wei Cheng

Department of Medical Science, Institute of Biotechnology, National Tsing Hua University, No. I0I, Section 2 Kuang-Fu Road, Hsinchu 30013, Taiwan

Tel +8863574 247I; +886357I 5934

Fax +88635742763

Email ytchou@life.nthu.edu.tw;

jwcheng@life.nthu.edu.tw
Purpose: Lung cancer and other solid tumors contain not only tumor cells but various types of stromal cells, such as fibroblasts and endothelial cells. In addition, tumors are infiltrated by inflammatory cells (neutrophils, macrophages, and lymphocytes). Tumor cells, stromal cells, and the tumor-associated leukocytes are responsible for the production of chemokines inside the tumor and the maintenance of systemic circulating chemokine levels. CXCL8 and its receptors, CXCR1 and CXCR2, were found to play important roles in tumor proliferation, migration, survival, and growth. We have developed a novel ELR-CXC chemokine antagonist CXCL8-IP10 based on the structure of CXCL8 and IP10.

Patients and methods: We assessed the anticancer efficacies of the blockade of CXCL8CXCR1/2 axis in the Lewis lung carcinoma (LL/2) model using CXCL8-IP10.

Results: We found that CXCL8-IP10 markedly reduced LL/2 cell anchorage-independent growth and invasion. Moreover, we demonstrated that CXCL8-IP10 could significantly suppress tumor growth and improve survival rate as well as lifespan of C57BL/6 mice inoculated with LL/2 cells.

Conclusion: Our results suggest that ELR-CXC chemokine antagonism would potentially be a useful therapeutic approach in patients with lung cancer.

Keywords: CXCL8, CXCR1, CXCR2, antagonist, Lewis lung carcinoma

\section{Introduction}

Cancer is now responsible for the majority of global health problems according to estimates from the World Health Organization (WHO) in 2018. ${ }^{1}$ Lung cancer accounts for the most significant proportion of diagnosed cancer $(11.6 \%$ of the total cases) and the mortality rate due to lung cancer has increased over the past years $\left(18.4 \%\right.$ of the total cancer deaths). ${ }^{1}$ There are only a few therapeutic options available for patients diagnosed with incurable advanced lung cancer. In addition to the marginally effective chemotherapies, the development of new, targeted agents such as epidermal growth factor receptor (EGFR) and anaplastic lymphoma receptor tyrosine Kinase (ALK) inhibitors have shown some promising results in its specific gene mutation population. ${ }^{2}$ However, more than $50 \%$ of the lung cancer patients in East Asia and $90 \%$ in the United States do not possess targetable mutations. Recent advances of immune checkpoint inhibitors (ICIs) for the treatment of advanced cancers, including non-small-cell lung cancer (NSCLC), also improved the response rate and prolonged long-term survival with limited adverse 
effects. $^{2}$ However, only $20-30 \%$ of the patients with advanced lung cancer can benefit from ICI treatments. Moreover, lung cancer cells harboring the epithelial-tomesenchymal transition (EMT) feature tend to develop tolerance to both targeted therapies and ICIs. ${ }^{3,4}$ Thus, novel therapeutic modalities targeting lung cancer cells with the EMT feature are urgently needed. Lewis lung carcinoma cell line (LL/2) used in this study was established from LLC1, and these cells have high tumorigenic ability in C57BL mice and induce primary tumors and lung metastases at a higher rate than the original tumor line (LLC1). ${ }^{5,6}$ Many research studies have established this cell line in immunocompetent mice as a model for NSCLC. ${ }^{7,8}$ This model reveals the immune tumor microenvironment in NSCLC and helps with understanding the response of associated therapies and potential drugs.

Chemokines are divided into $\mathrm{CXC}, \mathrm{CC}, \mathrm{C}$, and $\mathrm{CX} 3 \mathrm{C}$ subgroups. The $\mathrm{CXC}$ chemokines can be further classified into groups that have a Glu-Leu-Arg (ELR) motif and those groups without. The ELR-CXC chemokines are important for activating and trafficking inflammatory mediators through their engagement of the CXCR1 and/or CXCR2 receptors. ${ }^{9}$ CXCL8 (interleukin-8; IL-8), the prototypical ELR-CXC chemokine, is found to be involved in several diseases such as chronic obstructive pulmonary diseases (COPD), asthma, and cystic fibrosis. ${ }^{10}$ CXCR1 is the receptor for CXCL8 and CXCL6 (granulocyte chemoattractant protein-2) and CXCR2 binds CXCL8, CXCL6, and other ELR-CXC chemokines. CXCL8 binds with high affinity to both CXCR1 and CXCR2, while the others are relatively lower affinity ligands. ${ }^{9}$

Recently, the CXCL8-CXCR1/2 axis has been shown to play important roles in the tumorigenesis of solid tumors such as lung, colon, breast, prostate, pancreatic, melanoma, and ovarian cancers. ${ }^{11}$ CXCL8 can increase tumor growth, metastasis, and angiogenesis via upregulating MMP2 activity. ${ }^{12,13}$ Besides, CXCL8 secretion also increases anchorage-independent growth, proliferation, and invasion by regulating PI3K/Akt and Raf/MEK/ERK signaling. ${ }^{14}$ The roles of CXC chemokines and CXCR1/2 receptors in cancer have been depicted comprehensively in the review article. ${ }^{11}$ Basically, the CXCL8-CXCR1/2 signaling pathway was found to be involved in tumor progression, ${ }^{15-17}$ cancer stem cell formation, ${ }^{17}$ trafficking of myeloid-derived suppressor cells (MDSCs) into tumor microenvironment, ${ }^{18}$ and resistance to chemotherapeutic treatments. $^{19}$ Thus, the development of inhibitors and antibodies targeting the CXCL8-CXCR1/2 pathway provides an alternative therapeutic target for cancer.

Previously, we have developed a novel ELR-CXC chemokine antagonist CXCL8-IP10 based on the structure of CXCL8 and IP10. ${ }^{20}$ We have demonstrated that CXCL8IP10 markedly reduced Klebsiella-induced pulmonary inflammation, as assessed by gross pathology and histopathology, and airway neutrophil responses as well as airway and lung parenchymal myeloperoxidase, IL-1 $\beta$, IL-6, IL-10, IFN $\gamma$, and TNF- $\alpha$ levels. ${ }^{21}$ Herein, we study the anticancer efficacies of the blockade of CXCL8-CXCR $1 / 2$ axis in the LL/2 model using CXCL8-IP10. LL/2 cell line was chosen because it is the only reproducible syngeneic model for lung cancer with the EMT feature. ${ }^{22}$ Unlike the widely used xenograft models in which human lung cancer cells are implanted into mouse tissue, LL/2 cells are immunologically compatible with the murine system and the tumor microenvironment can be fully produced. The true immune and toxicity responses due to the blockade of CXCL8-CXCR1/2 axis by CXCL8-IP10 can then be measured accordingly.

\section{Materials and methods Cell line and culture conditions}

$\mathrm{LL} / 2$ is a cell line established from the lung of a C57BL mouse bearing a tumor due to an implantation of primary Lewis lung carcinoma. ${ }^{5}$ We obtained this cell line from Bioresource Collection and Research Center, Taiwan. These lung cancer cell lines were grown in Dulbecco's Modified Eagle's Medium (DMEM, Gibco ${ }^{\mathrm{TM}}$ ) with fetal bovine serum (FBS, Biological industries) to a final concentration of $10 \%$.

\section{CXCL8-survival and CXCL8-EMT analyses in human lung cancer data sets}

The TCGA LUAD data sets were downloaded from the UCSC Cancer Genomics Browser. CXCL8 was used for analyses of gene expression with overall survival and recurrence-free survival. High and low expression groups were split by Cutoff Finder Web for plotting KaplanMeier curves. Analysis of the CXCL8 correlation between $\mathrm{CDH} 2$ and between VIM indicates CXCL8 is positively associated with mesenchymal feature in lung adenocarcinoma patients from the UCSC Cancer Genomics Browser. By contrast, correlation between CDH1 and CXCL8 gene expression indicates negative association in epithelial characteristics. 


\section{Western blot analysis}

$\mathrm{LL} / 2$ were plated in $100-\mathrm{mm}$ dish at $1 \times 10^{6}$ cells and cultured for $24 \mathrm{hrs}$ in growth medium. After $24 \mathrm{hrs}$, cells were treated with CXCL8-IP10 for $48 \mathrm{hrs}$. The cells were harvested and added lysis buffer plus Phosphatase Inhibitor Cocktail (GBioscience). The protein concentration was determined by Bradford Protein Assay Kit (GeneMark). Each sample (60 $\mathrm{mg}$ ) was loaded in $8 \%$ SDS-PAGE and was blotted onto a 0.45 Micron PVDF transfer membrane (GVS North America). Primary antibodies, N-cadherin (GeneTex) and E-cadherin (GeneTex), were diluted in 1x PBS containing $0.1 \%$ Tween ${ }^{\circledR} 20$ (PBST) and incubated at $4^{\circ} \mathrm{C}$ overnight. After washing with PBST, the membranes were incubated with Anti-Rabbit IgG (GeneTex) secondary antibody for $1 \mathrm{hr}$. The signals were detected by ImageQuant LAS $4000 \mathrm{mini}$ luminescent image analyzer (GE Healthcare Bio-Sciences $A B)$. The protein bands were quantified using ImageJ software to determine the integrated density of each band, and the ratio to GAPDH was then calculated. The experiments were repeated at least 3 times.

\section{Doubling-time assays of suspended cells} LL/2 were plated in non-coated 24 -well plates at $2 \times 10^{4}$ cells/well and cultured for $72 \mathrm{hrs}$ in growth medium. Cells were treated with CXCL8, CXCL8 plus CXCL8-IP10, or CXCL8-IP10. Measurements were followed up in a previous study. ${ }^{23}$ Cells were counted from at least 3 wells of the same group in a single experiment. After $72 \mathrm{hrs}, 3$ counting results were analyzed to get the doubling-time of cells. The experiments were repeated at least 3 times.

\section{Cell anchorage-independent growth assays}

Cell proliferation assay was performed using the cell counting kit-8 (CCK-8) (Dojindo, Kumamoto, Japan). LL/2 cells were plated in non-coated 24 -well plates at $5 \times 10^{4}$ cells/well and cultured in growth medium. Cells were treated with CXCL8, CXCL8 plus CXCL8-IP10, or CXCL8-IP10. Equal volume of growth medium was added in the control wells. Assays were manipulated according to the operation manual. The absorbance of each well was determined at 450 $\mathrm{nm}$ and cell proliferation rate was calculated. These experiments were repeated at least 3 times.

\section{Cell cycle assays}

Cell cycle assays of cancer cells were evaluated by propidium iodide (PI) as reported previously. ${ }^{24} \mathrm{LL} / 2$ cells $\left(1 \times 10^{5}\right.$ cells per well) were cultured in 6-well dishes serum-free for 16-18 hrs, then treated without (control) or with CXCL8, CXCL8 plus CXCL8-IP10, or CXCL8IP10 for $24 \mathrm{hrs}$. The results were followed by an analysis with a flow cytometer (BD Accuri ${ }^{\mathrm{TM}}$ C6) and FlowJo 7.6.1 for data acquisition and analysis. The assays were repeated at least 3 times.

\section{In vitro invasion assays of LL/2}

The invasion ability of LL/2 was performed using $8 \mu \mathrm{m}$ pore size transwell (Corning FluoroBlok ${ }^{\mathrm{TM}}$ ). ${ }^{25}$ The assay was executed in accordance with the previous research. ${ }^{26}$ A total of $2.5 \times 10^{4}$ cells in DMEM medium containing $1 \%$ FBS were seeded into the upper chamber, and then treated with the target drug (CXCL8, CXCL8-IP10 or MIP-2). DMEM medium containing $10 \%$ FBS was added into the lower chamber. After 24-hr incubation, the invasive cells in the bottom of the membrane were fixed, imaged, and counted using a fluorescence microscope at 100x magnification over 5 random fields in each well. The invasive experiments were repeated at least 3 times.

\section{Anti-tumor effects in vivo studies}

Male C57BL/6 mice (4- to 6-week old) were purchased from the National Laboratory Animal Center (Taipei, Taiwan) and maintained in a specific pathogen-free and controlled environment. LL/2 cells were harvested and resuspended at a density of $1 \times 10^{7}$ cells $/ \mathrm{mL}$ in sterile phosphate-buffered saline. In the experimental group, LL/2 cells were pre-treated with $200 \mu \mathrm{g} / \mathrm{mL}$ of CXCL8IP10. A total of $1 \times 10^{6}$ cells (mixed equal volume of matrigel) were injected subcutaneously into the back of the mice (6/group). Then, CXCL8-IP10 (500 $\mu \mathrm{g} / \mathrm{kg})$ or saline (control group) was administered by intraperitoneal injection 3 times a week, and the tumor size was measured on the same day. The volume of the tumors was calculated using the following formula: Tumor size $\left(\mathrm{mm}^{3}\right)=0.5 \times$ (width ${ }^{2} \times$ length). The results were shown as volume \pm SEM. The survival rates of tumor-bearing mice were observed and recorded continuously until all animals were dead. All the procedures were conducted according to the regulations of Laboratory Animal Care and Use Committee or Group Setup and Management and the law of Animal Protection. The study was approved by the Ethics Committee of National Tsing Hua University.

\section{Statistical analyses}

All results are presented as means \pm SEM. Levels of significance were calculated using one-way ANOVA, followed by 
Student's $t$-tests (two-tailed), using GraphPad Prism software (significance between data with a threshold of $\left.{ }^{*} P<0.05 ; * * P<0.01 ; * * * P<0.001\right)$.

\section{Results}

\section{CXCL8 plays a vital role in cancer survival and EMT in human lung cancer data sets}

Previous studies have shown that CXCL8 promotes cell progression and EMT in breast cancer, lung cancer, and pancreatic cancer. ${ }^{27}$ Besides, reduced levels of CXCL8 and inhibition of CXCR2 prolong patient overall survival. ${ }^{11}$ Big Data analysis indicated that CXCL8 is highly associated with patients' overall survival and progression-free survival (Figure 1A, Table 1). In addition, the mesenchymal markers $\mathrm{CDH} 2$ and VIM show a positive correlation with CXCL8; by contrast, the epithelial marker CDH1 shows a negative correlation with CXCL8 (Figure 1B). Taking these Big Data analysis together, we found that CXCL8 indeed actuates EMT and further impacts survival in clinical practice.

\section{CXCL8-IPI0 inhibit EMT characteristics of cancer cells}

According to clinical data analysis, we realize EMT has high correlation with CXCL8. Besides, in vivo research also indicates the essential role of CXCL8 signaling for the acquired mesenchymal and invasive features of tumor cells. Moreover, it suggests that CXCL8 signaling blockade may act as a means of targeting mesenchymal-like and invasive tumor cells. ${ }^{27}$ To confirm that CXCL8-IP10 can compete with CXCL8 and inhibit EMT, Western blot analysis of endothelial and mesenchymal marker, E-cadherin and N-cadherin, was implemented. The results show CXCL8-IP10 can significantly reduce EMT by enhancing E-cadherin expression and decreasing N-cadherin expression compared to control panel (Figure 2A-C). These data give us confidence to ensure CXCL8-IP10 can inhibit CXCL8-induced cancer characteristics in vivo experiences.

\section{CXCL8-IPI0 decreases the anoikis-} resistant growth rate of cancer cells

LL/2 is a highly malignant tumorigenic cell line with the EMT feature (slender phenotype) derived from C57BL mice. Similar to the characteristics of human lung cancer cells, it divides relentlessly and grows abnormally rapid. ${ }^{5}$ To test whether CXCL8 induction promotes anoikis resistance of LL/2, we measured the doubling time of LL/2 under a suspended condition in the presence and absence of CXCL8 stimulation. In the doubling-time assay, we found that CXCL8-IP10 can significantly increase the doubling time

A
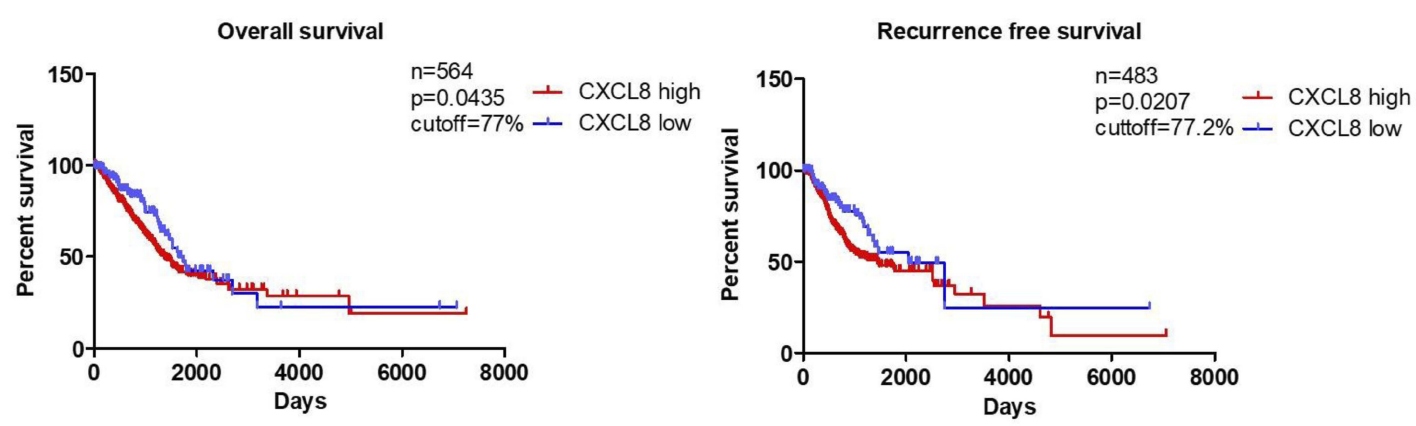

B
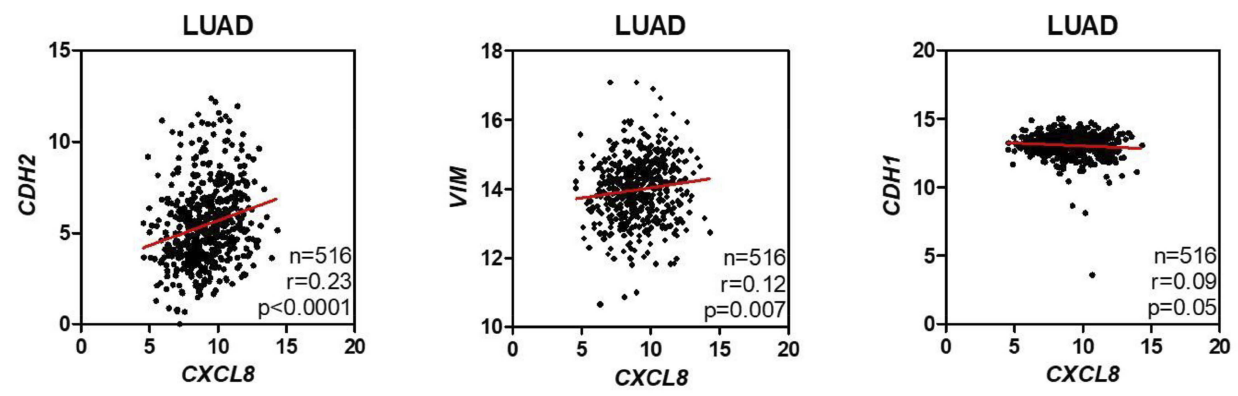

Figure I CXCL8-survival and CXCL8-EMT clinical analysis by the TCGA LUAD from the UCSC Cancer Genomics Browser.

Notes: (A) CXCL8 expression predicts poor prognosis in lung adenocarcinoma. (B) CXCL8 level is associated with EMT marker expression in lung adenocarcinoma. 
Table I CXCL8 expression and outcome of survival

\begin{tabular}{|c|c|c|c|c|}
\hline \multirow[t]{2}{*}{ Variables } & & \multicolumn{3}{|c|}{ CXCL8 mRNA expression } \\
\hline & & High $(n=4 \mid 9)$ & Low $(n=145)$ & $P$-value \\
\hline \multirow[t]{2}{*}{ os } & $\begin{array}{l}\text { Md } \\
95 \% \mathrm{Cl}\end{array}$ & $\begin{array}{l}22.20 \\
27.52-32.99\end{array}$ & $\begin{array}{l}23.03 \\
27.68-38.25\end{array}$ & 0.0435 \\
\hline & & High $(n=359)$ & Low $(n=124)$ & p-value \\
\hline RFS & $\begin{array}{l}\text { Md } \\
95 \% \mathrm{Cl}\end{array}$ & $\begin{array}{l}\mid 8.87 \\
24.04-29.61\end{array}$ & $\begin{array}{l}20.95 \\
23.37-32.82\end{array}$ & 0.0207 \\
\hline
\end{tabular}

Notes: The cutoff value of OS and RFS is $77 \%$ and $77.2 \%$, respectively. The unit of $\mathrm{Md}$ and $95 \% \mathrm{Cl}$ are months.

Abbreviations: OS, overall survival; RFS, recurrence-free survival; Md, median; $\mathrm{Cl}$, confidence interval.

A

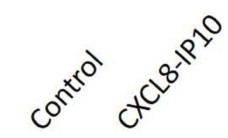

E-cadherin

$\mathrm{N}$-cadherin

GAPDH

B

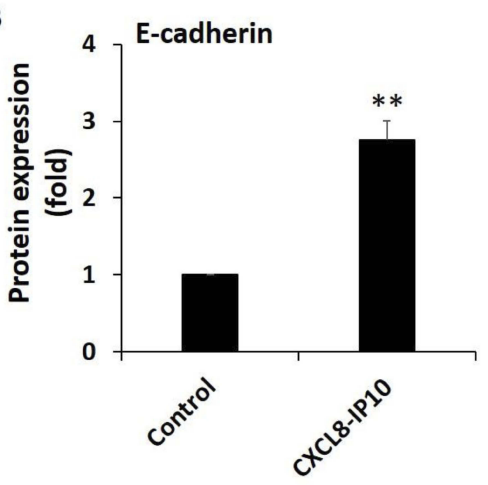

C

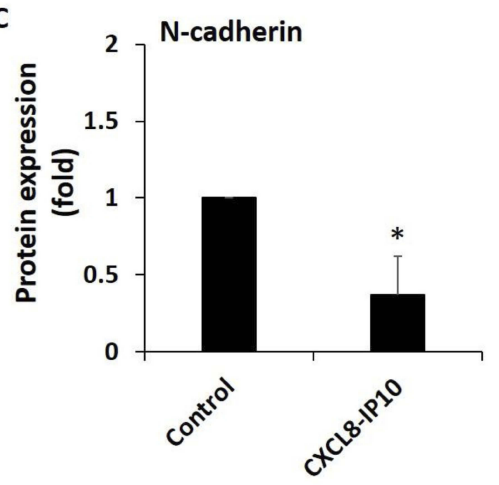

Figure 2 CXCL8-IPI0 inhibit the EMT characteristics of LL/2.

Notes: After treating CXCL8-IPI $0200 \mathrm{ng} / \mathrm{mL}$ for 48 hrs, (A) Western blot analysis of endothelial, E-cadherin, and mesenchymal marker, N-cadherin, was conducted. (B) and (C) The panels of Western blot analysis were calculated and quantified into bar chart. Results were obtained from three experiments, and the bars represent means $\pm S E M$. $* P<0.05$; $* * P<0.0$ I, Student's $t$-test.

of LL/2 compared with the control (Figure 3A). On the other hand, we found that CXCL8 reduced the doubling time of LL/2 (Figure 3B). The addition of CXCL8-IP10 with CXCL8 was found to increase the doubling-time again (Figure 3B). This result indicates that CXCL8-IP10 can slow down the anoikis-resistant growth rate of cancer cells.

\section{CXCL8-IPI0 significantly inhibits cancer cells anchorage-independent growth}

Previous studies have shown that CXCL8 is an autocrine growth factor in lung cancer cells. ${ }^{28}$ Existence of CXCL8 resulted in a greater potential for anchorage-independent growth. ${ }^{19}$ To assess whether CXCL8-IP10 can efficiently block the CXCL8-induced anchorage-independent growth, we implemented cell suspended growth by CCK8 assay in $24 \mathrm{hrs}$. The results showed that CXCL8 indeed significantly increased the anchorage-independent rate in a short period (Figure 4B), but when CXCL8-IP10 was present, the growth rate all notably decreased (Figure 4A and B).

\section{Cell cycle GI phase was arrested by CXCL8-IPIO}

After realizing CXCL8-IP10 can decrease cancer rapid anoikis-resistant and anchorage-independent growth characteristics, we would like to understand how it affects cells in more detail. Many studies in cancer research showed 


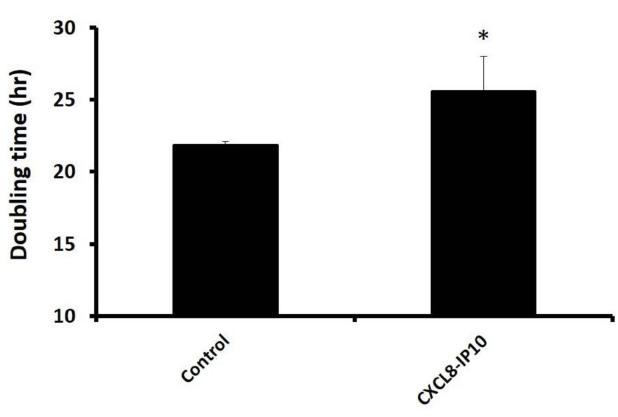

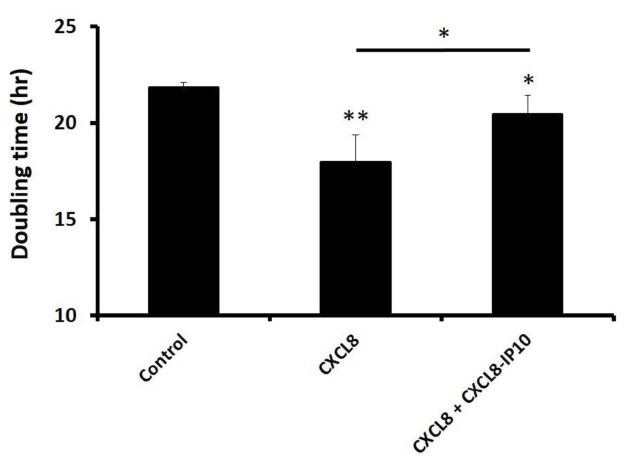

Figure 3 Inhibition of CXCL8-induced anoikis-resistant growth by CXCL8-IPI0 in the doubling-time assay of suspended LL/2 cells.

Notes: The cells were seeded in non-coated 24-well plates and cultured for $72 \mathrm{hrs}$ in DMEM and were counted every 24 hrs by pipetting $10 \mu \mathrm{L} /$ well to a hemocytometer using the dye exclusion test. (A) Cells were treated with CXCL8-IPI0 $200 \mathrm{ng} / \mathrm{mL}$ and compared to control (DMEM). (B) Cells were treated with CXCL8 I00 ng/mL or CXCL8 $100 \mathrm{ng} / \mathrm{mL}$ plus CXCL8-IPI0 $200 \mathrm{ng} / \mathrm{mL}$. Results were obtained from three experiments, and the bars represent means $\pm S E M$. $* P<0.05$; $* * P<0.0 \mathrm{I}$, Student's $t$-test.

A

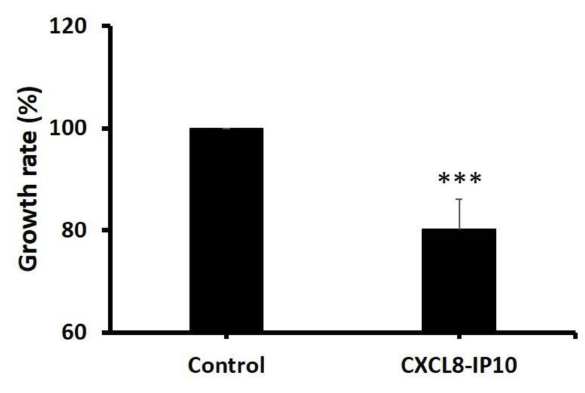

B

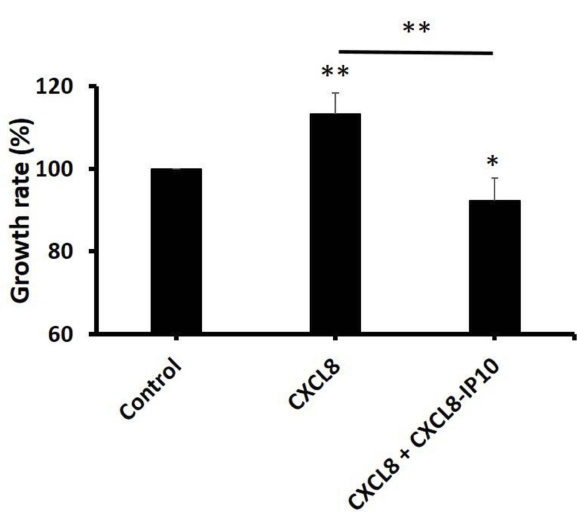

Figure 4 Cell anchorage-independent growth decreased when treated with CXCL8-IPI0.

Notes: LL/2 cells were plated in non-coated 24-well plates and cultured in growth medium. After 24 hrs, the media with cells were taken from wells to the other 96 -well plates, and CCK-8 solution was added about $2 \mathrm{hrs}$. The absorbance of each well was determined at $450 \mathrm{~nm}$ and cell growth rate was calculated. (A) Cells were treated with CXCL8-IPI0 $200 \mathrm{ng} / \mathrm{mL}$ and compared to control (DMEM). (B) Cells were treated with CXCL8 $100 \mathrm{ng} / \mathrm{mL}$ or CXCL8 $100 \mathrm{ng} / \mathrm{mL}$ plus CXCL8-IPI0 200 ng/mL. Results were obtained from three experiments, and the bars represent means $\pm S E M$. $* P<0.05 ; * * P<0.01 ; * * * P<0.00 I$, Student's $t$-test.

that induced G1 phase cell cycle arrestment is associated with apoptosis and a decrease in proliferation. ${ }^{29-31}$ Furthermore, studies indicated that G1 phase arrest was accompanied by a decreased number of cells in S and G2 phase. $^{24,32}$ In the cell cycle assay studied by flow cytometry, it showed a significant decrease of the G2 phase and an increase of the G1 phase by addition of CXCL8-IP10 (Figure 5A). This result again proved that CXCL8-IP10 can inhibit LL/2's growth. Increasing cell percentage in G1 phase and decreasing cell percentage in $\mathrm{S}$ phase were found in the CXCL8 plus CXCL8-IP10 group while compared with the CXCL8 only group (Figure 5B). This result suggested that cells stayed in G1 phase and reduced synthesis of protein for mitosis in G2 phase when treated with CXCL8-IP10. In the CXCL8-IP10 treatment group, a high possibility of cell apoptosis or slower growth was observed.

\section{CXCL8-IPI0 decreased cell invasion while CXCL8 and MIP-2 induced cell invasion}

The metastasis of tumor cells is the main reason for the progressive deterioration and development of cancer. ${ }^{33}$ The expression of CXCL8 and its receptors CXCR $1 / 2$ play critical roles in cell migration and invasion in many types of cancer. ${ }^{34}$ CXCL8 secretion increases anchorageindependent growth and accompanies malignant behavior, including angiogenesis, adhesion, and invasion. ${ }^{14}$ Matrigel-coated transwell assay was used to investigate the effect of CXCL8-IP10 on LL/2's invasion ability. 


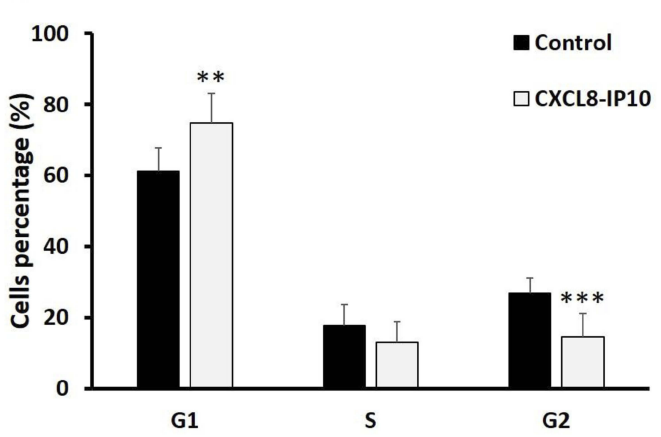

B

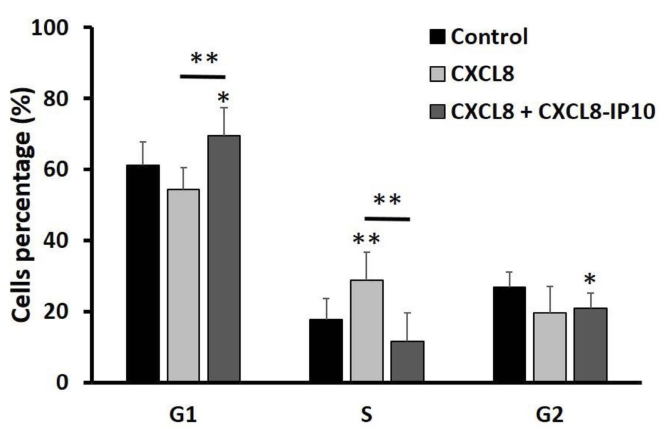

Figure 5 GI Cell cycle arrest by CXCL8-IPIO on suspended LL/2 cells.

Notes: The cells were starved for $16-18 \mathrm{hrs}$, and then treated with drugs for $24 \mathrm{hrs}$. The results were followed by analysis with a flow cytometer (BD Accuri ${ }^{\mathrm{TM}} \mathrm{C} 6$ ). Cell cycle analysis of (A) CXCL8-IPI0 $200 \mathrm{ng} / \mathrm{mL}$ compared with control (growth medium) and (B) CXCL8 I00 ng/mL plus CXCL8-IPI0 200 ng/mL compared with CXCL8 I00 $\mathrm{ng} / \mathrm{mL}$ shows arrest in $\mathrm{GI}$ phase. Results were obtained from at least three experiments, and the bars represent means $\pm S E M$. $* P<0.05$; $* * P<0.0 \mathrm{I}$; $* * * P<0.00 \mathrm{I}, \mathrm{Student}$ 's $t$-test.

The results showed that CXCL8 excited LL/2's invasion ability and, on the other hand, CXCL8-IP10 significantly suppressed CXCL8-stimulated cell invasion (Figure 6A). As shown in the CXCL8 stimulation assay, MIP-2, the functional homologue of CXCL8 in murine, demonstrated the same results in the MIP-2-induced cell invasion (Figure 6B). CXCL8-IP10 also decreased the invasion rate in the MIP-2 stimulated group. These results suggested that not only can CXCL8-IP10 attenuate CXCL8induced invasion but also attenuate the MIP-2-induced invasion in murine.

\section{CXCL8-IPI0 inhibits tumor growth and prolongs the survival in C57BL/6 mice}

To evaluate the effect of CXCL8-IP10 in vivo, we used the LL/2 allogeneic model in C57BL/6 mice. Mice were inoculated with LL/2 cells subcutaneously and then treated with saline or CXCL8-IP10 (500 $\mu \mathrm{g} / \mathrm{kg})$ by intraperitoneal (i.p.) injection 3 times a week. We found that CXCL8-IP10 significantly inhibited tumor growth in vivo compared to the saline control group (Figure 7A and C). Additionally, treatment of CXCL8-IP10 did not lead to a reduction in the body weight of mice (data not shown), meaning that CXCL8IP10 might have no toxicity effect in vivo. Furthermore, CXCL8-IP10 prolonged the lifespan of tumor-bearing mice (Figure 7B). The median lethal time (LT50) of CXCL8IP10 group and control group was 36 days and 28 days, respectively. These results demonstrated that CXCL8-IP10 could significantly suppress tumor growth and improve the survival rate of $\mathrm{C} 57 \mathrm{BL} / 6$ mice; however, there was no significant metastasis nodules occurrence in this model
(Figure 7D), which may have been due to the low incidence of metastasis of tumors by subcutaneous injection.

\section{Discussion}

Lung cancer is the leading cause of cancer deaths in most countries. ${ }^{1}$ The development and implementation of novel therapeutics based on new, targeted approaches are urgently required. Lung cancer and other solid tumors contain not only tumor cells but various types of stromal cells, such as fibroblasts and endothelial cells. In addition, tumors are infiltrated by inflammatory cells (neutrophils, macrophages, and lymphocytes). ${ }^{35}$ Tumor cells, stromal cells, and the tumor-associated leukocytes are responsible for the production of chemokines inside the tumor and systemic circulating chemokine levels. CXCL8 and its receptors, CXCR1 and CXCR2, were found to play important roles in tumor proliferation, migration, survival, and growth. ${ }^{11}$ The impact of CXCR1 and CXCR2 on NSCLC in vitro and in vivo was studied recently. ${ }^{24}$ Concretely speaking, Luppi et al showed that the CXCL8 mediated the proliferation of NSCLC by additional CXCL8 ${ }^{36}$ and Wang $\mathrm{Y}$ et al demonstrated that overexpressing-CXCL8 cell lines show increased anchorage-independent growth but decreased in IL-8 depleted cell lines. ${ }^{14}$ Moreover, the role of CXCL8 in the resistance of lung cancer cells to EGFR inhibitors was reported. ${ }^{19}$ However, the above-mentioned results were all studied by using xenograft mouse models that may not represent the true tumor microenvironment in patients with lung cancer.

Chemokines are relatively small molecular weight proteins (8-10 Kd) that are important in the recruitment of leukocytes to extravascular tissues. ${ }^{9}$ The ELR $^{+}$CXC chemokines include 

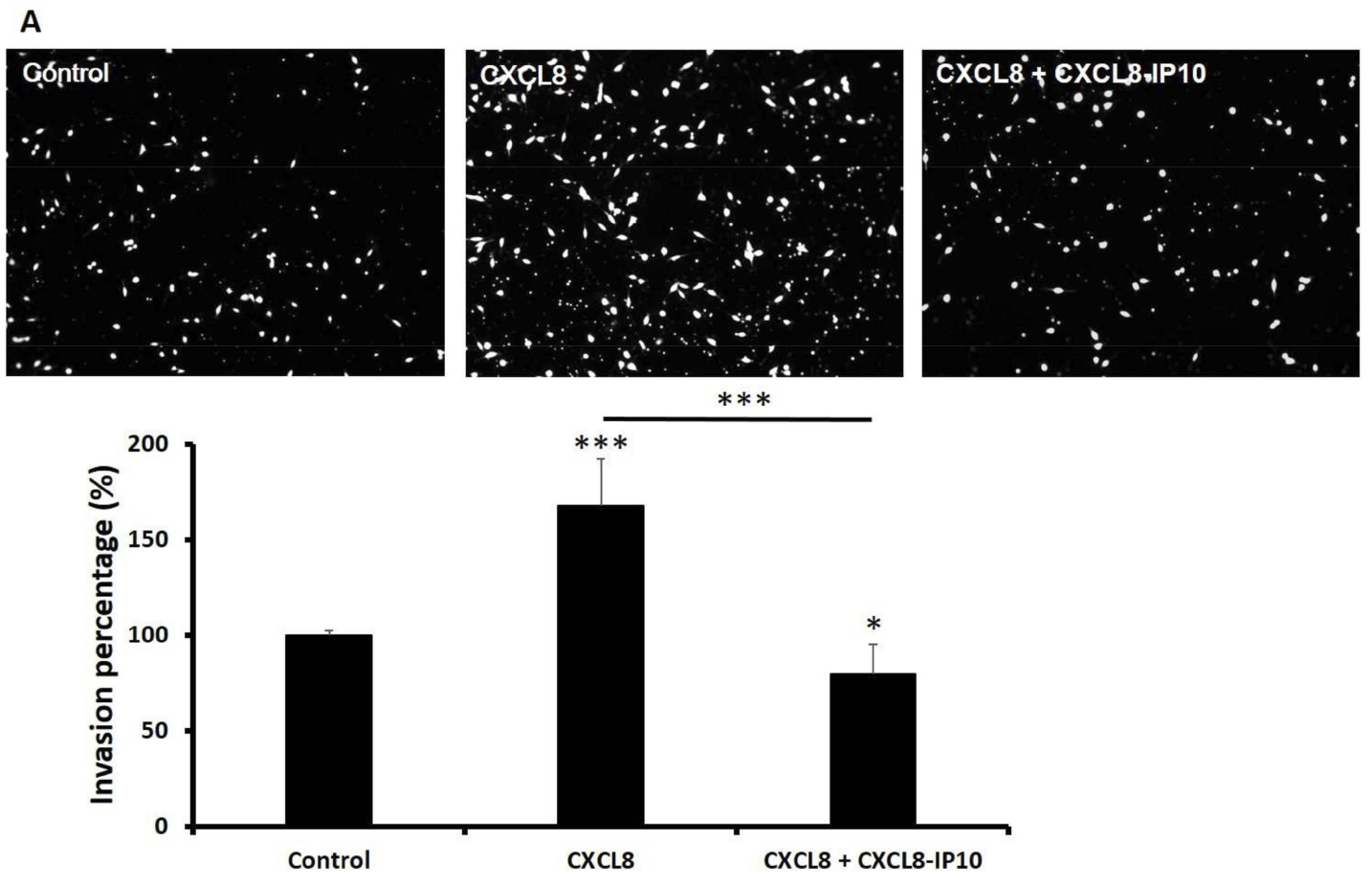

\section{B}
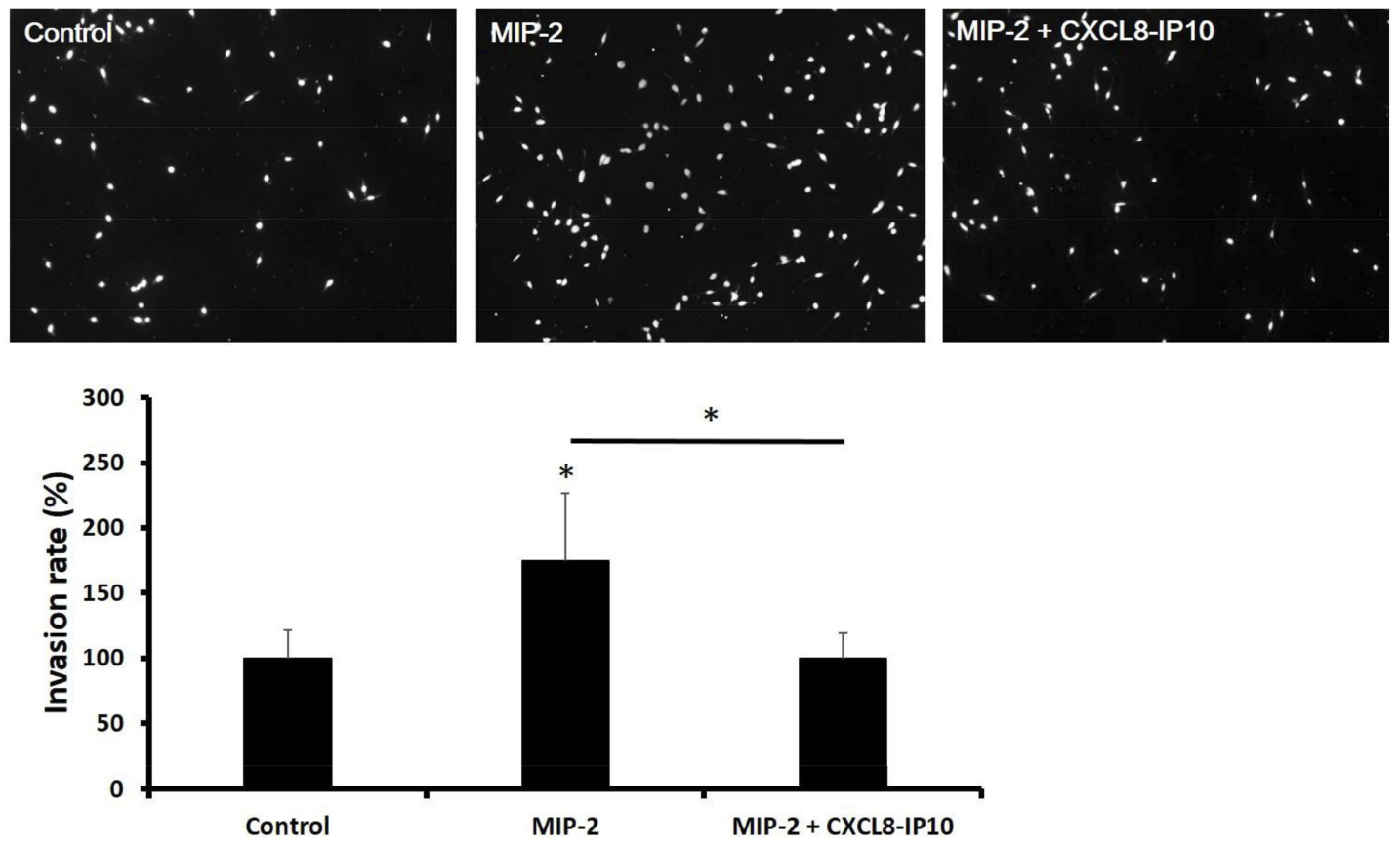

Figure 6 The CXCL8 and MIP-2 capability of invasion was attenuated by CXCL8-IPI0 in LL/2 cells.

Notes: Cells were seeded onto Matrigel-coated polycarbonate filters to analyze their invasive potentials. The cells were then incubated for 24 hrs in modified Boyden chambers and the cells that invaded through the filters were stained by PI and counted under a light microscope. Representative fields of invasive cells on the transwell membrane (at 100x magnification) and the invasion rate show in two conditions. (A) cells were treated with CXCL8 I00 ng/mL or CXCL8 I00 ng/mL plus CXCL8-IPI0 200 $\mathrm{ng} / \mathrm{mL}$ and (B) cells were treated with MIP-2 $50 \mathrm{ng} / \mathrm{mL}$ or MIP-2 $50 \mathrm{ng} / \mathrm{mL}$ plus CXCL8-IPI0 $200 \mathrm{ng} / \mathrm{mL}$. Results were obtained from five random fields in each well and were repeated three times. The bars represent means \pm SEM. $* P<0.05$; $* * * P<0.001$, Student's $t$-test.

CXCL1, CXCL2, CXCL3, CXCL5, CXCL6, CXCL7, and CXCL8. ${ }^{9}$ These redundantly expressed chemokines all bind to either the CXCR1 or CXCR2, and collectively recruit neutrophils into the local inflammatory responses ${ }^{9}$ in a complex multistep process. ${ }^{37-39}$ We have previously designed CXCL8-IP10, a very high-affinity antagonist of CXCR1 and CXCR2, based on the structures of CXCL8 and IP10. ${ }^{21}$ CXCL8-IP10 has a structural frame of CXCL8 and the 30's 
A

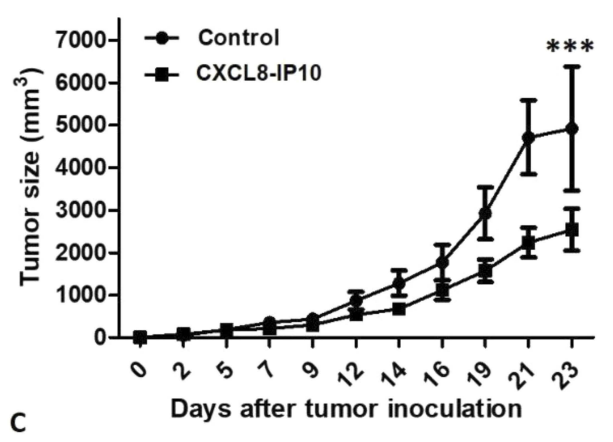

C

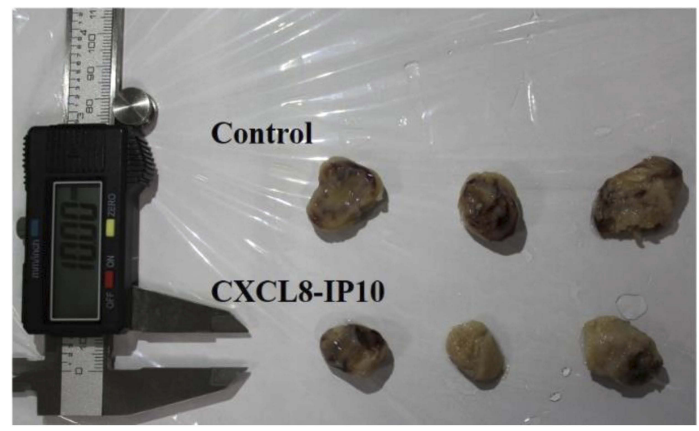

B

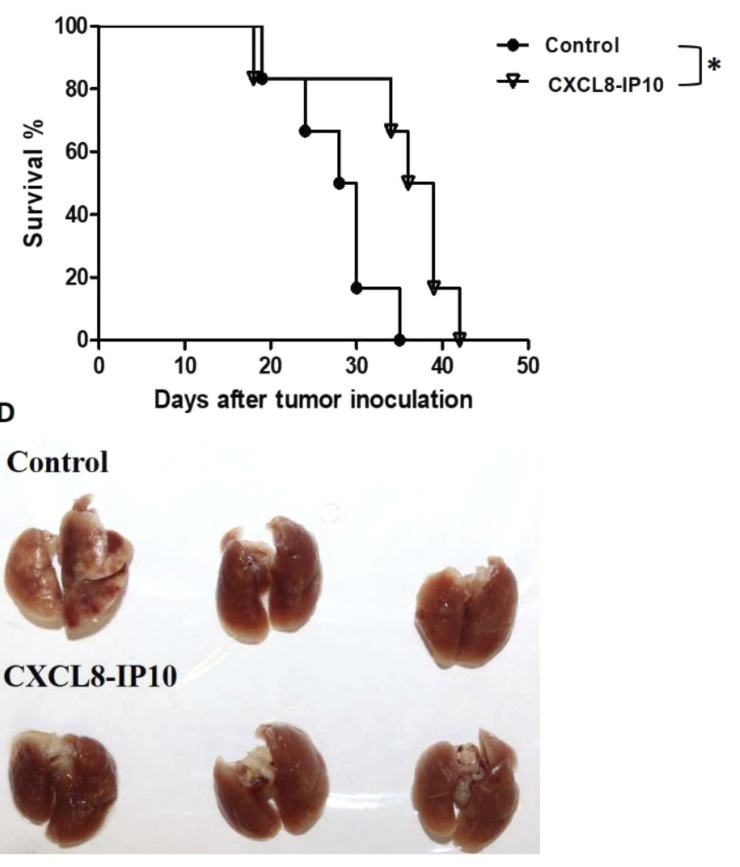

Figure 7 In vivo anti-tumor effect of CXCL8-IPI0.

Notes: LL/2 cells were subcutaneously inoculated to male C57BL/6 mice (4- to 6-week old). Then, CXCL8-IPI0 (500 $\mu \mathrm{g} / \mathrm{kg})$ or saline (control group) was administrated by intraperitoneal injection every 3 times a week. (A) The LL/2 tumor size was measured and (B) the survival curves of tumor-bearing mice were recorded after being treated with CXCL8-IPI0 and control (saline). The survival rates of tumor-bearing mice were observed and recorded continuously until all mice were dead. (C) and (D) After mice were dead, tumor and lung tissue were taken out and pictures were taken as shown. Data are shown as means $\pm S E M(n=6)$. $* P<0.05$; $* * * P<0.00 \mathrm{I}$ compared with control group, Student's $t$-test.

loop of CXCL10 benefited from strong receptor-binding affinity but not the neutrophil-attracting properties. ${ }^{21}$ CXCL8IP10 effectively antagonizes human neutrophil responses to ELR-CXC chemokine CXCL8. ${ }^{21}$ Moreover, CXCL8-IP10 was found to efficiently block pulmonary pathology in a mouse model of Klebsiella pneumoniae infection. ${ }^{21}$

In this study, we used the Lewis lung carcinoma (LL/2) model to investigate the impact of CXCR $1 / 2$ antagonism by CXCL8-IP10. Lewis lung carcinoma was chosen because it is immunologically compatible with the murine system and the tumor microenvironment can be fully produced; furthermore, it is normally established to reveal the NSCLC in immunocompetent mice. ${ }^{7,8}$ Besides, LL/2 has an EMT feature which indicated as a driving force in tumor progression and trigger cancer cells to evade their original place and to colonize other locations, cell lines have this feature may be a malignant cancer. ${ }^{40}$ In this study, we did not' discuss about EMT in depth, but EMT shows positive correlation with CXCL8. ${ }^{41}$ Furthermore, CXCL8-IP10 significantly decreased the N-cadherin expression (Figure 2C). The addition of CXCL8-IP10 with CXCL8 significantly increased the doubling-time, hence slowed down the growth rate of LL/2 cells. One thing needed to be mentioned is that in the doubling-time assay and the CCK8 assay, the LL/2 cells were cultured in suspended conditions to mimic the metastasis growth, anoikis-resistant growth condition of cancer cells. Previous studies have shown that the increasing arrest in the G1 phase will reduce cell proliferation and enhance cell apoptosis. ${ }^{29-31}$ Our results also indicate that CXCL8IP10 increased the LL/2 cell arrest in G1. These results were verified again by the CXCL8-IP10's ability to inhibit cancer growth. The novel CXCL8-IP10 effected signal pathway is still unclear, but in previous studies, JAK2/ STAT3, PI3K/AKT, and Raf/MEK/ERK pathways are normally involved in CXCL8-associated-EMT, ${ }^{41}$ proliferation/survival ${ }^{42,43}$, and drug resistance. ${ }^{44}$

Other than the in vitro studies, the in vivo pharmacology effects of CXCL8-IP10 on C57BL/6 mice inoculated with LL/ 2 tumor cells showed CXCL8-IP10 significantly inhibited the tumor growth in vivo without disrupting diet consumption when compared to the PBS control group (data not shown). Additionally, treatment of CXCL8-IP10 did not lead to a reduction in the body weight of mice which indicated that CXCL8-IP10 might not have adverse effects. Furthermore, CXCL8-IP10 prolonged the lifespan of tumor-bearing mice. 
The median lethal time (LT50) of the CXCL8-IP10 group and control group was 36 days and 28 days, respectively. The overall results demonstrated that CXCL8-IP10 could significantly suppress tumor growth and improve the survival rate of C57BL/6 mice.

\section{Conclusion}

Our data indicate that the ELR-CXC antagonist CXCL8IP10 can effectively decrease tumor cell anoikis-resistant and anchorage-independent growth by G1 phase arrest, invasion, as well as reduce tumor size and prolong the lifespan of tumor-bearing mice in the Lewis lung carcinoma model. It thereby suggests that the ELR-CXC chemokine antagonism would potentially be a useful therapeutic approach in patients with lung cancer.

\section{Acknowledgment}

We thank Justin Cheng and Daniel Cheng for editing the manuscript.

\section{Disclosure}

We report the industrial-academic collaboration grant from Rise Biopharmaceuticals Inc. The authors report no other conflicts of interest in this work.

\section{References}

1. Bray F, Ferlay J, Soerjomataram I, Siegel RL, Torre LA, Jemal A. Global cancer statistics 2018: GLOBOCAN estimates of incidence and mortality worldwide for 36 cancers in 185 countries. CA Cancer J Clin. 2018;68:394-424. doi:10.3322/caac.21492

2. Dong J, Li B, Lin D, Zhou Q, Huang D. Advances in targeted therapy and immunotherapy for non-small cell lung cancer based on accurate molecular typing. Front Pharmacol. 2019;10:230. doi:10.3389/ fphar.2019.00230

3. Mak MP, Tong P, Diao L, et al. A patient-derived, pan-cancer EMT signature identifies global molecular alterations and immune target enrichment following epithelial-to-mesenchymal transition. Clin Cancer Res. 2016;22(3):609-620. doi:10.1158/1078-0432.CCR-15-0876

4. Poh ME, Liam CK, Rajadurai P, Chai CS. Epithelial-to-mesenchymal transition (EMT) causing acquired resistance to afatinib in a patient with epidermal growth factor receptor (EGFR)-mutant lung adenocarcinoma. J Thorac Dis. 2018;10(7):E560-E563. doi:10.21037/ jtd.2018.06.122

5. Bertram JS, Janik P. Establishment of a cloned line of Lewis Lung Carcinoma cells adapted to cell culture. Cancer Lett. 1980;11(1):6373. doi:10.1016/0304-3835(80)90130-5

6. Lewis MR, Aptekman PM. Atrophy of tumors caused by strangulation and accompanied by development of tumor immunity in rats. Cancer. 1952;5 (2):411-413. doi:10.1002/1097-0142(195203)5:2<411::aid-cncr2820050 233>3.0.co;2-r

7. Schoenhals JE, Seyedin SN, Anderson C, et al. Uncovering the immune tumor microenvironment in non-small cell lung cancer to understand response rates to checkpoint blockade and radiation. Transl Lung Cancer R. 2017;6(2):148-158. doi:10.21037/tlcr.2017. 03.06
8. Weeratna RD, Bourne LL, Sullivan SM, Davis HL, Krieg AM. Combination of a new TLR9 agonist immunomodulator (CpG 7909) and paclitaxel for treatment of metastatic Lewis Lung Carcinoma (LLC). J Clin Oncol. 2004;22(14):702s-702s. doi:10.1200/jco.2004.22.14_suppl.7346

9. Baggiolini M. Chemokines in pathology and medicine. J Intern Med. 2001;250:91-104.

10. Aggarwal BB, Shishodia S, Sandur SK, Pandey MK, Sethi G. Inflammation and cancer: how hot is the link? Biochem Pharmacol. 2006;72:1605-1621. doi:10.1016/j.bcp.2006.06.029

11. Ha H, Debnath B, Neamati N. Role of the CXCL8-CXCR1/2 axis in cancer and inflammatory diseases. Theranostics. 2017;7:1543-1588. doi: $10.7150 /$ thno. 15625

12. Huang SY, Mills L, Mian B, et al. Fully humanized neutralizing antibodies to interleukin-8 (ABX-IL8) inhibit angiogenesis, tumor growth, and metastasis of human melanoma. Am $J$ Pathol. 2002;161(1):125-134. doi:10.1016/S0002-9440(10)64164-8

13. Luca M, Huang SY, Gershenwald JE, Singh RK, Reich R, BarEli M. Expression of interleukin- 8 by human melanoma cells up-regulates MMP-2 activity and increases tumor growth and metastasis. Am J Pathol. 1997;151(4):1105-1113.

14. Wang Y, Xu RC, Zhang XL, et al. Interleukin-8 secretion by ovarian cancer cells increases anchorage-independent growth, proliferation, angiogenic potential, adhesion and invasion. Cytokine. 2012;59 (1):145-155. doi:10.1016/j.cyto.2012.04.013

15. Keane MP, Belperio JA, Xue YY, Burdick MD, Strieter RM. Depletion of CXCR2 inhibits tumor growth and angiogenesis in a murine model of lung cancer. J Immunol. 2004;172(5):2853-2860. doi:10.4049/jimmunol.172.5.2853

16. Steele CW, Karim SA, Leach JDG, et al. CXCR2 inhibition profoundly suppresses metastases and augments immunotherapy in pancreatic ductal adenocarcinoma. Cancer Cell. 2016;29(6):832-845. doi:10.1016/j.ccell.2016.04.014

17. Chen LY, Fan J, Chen H, et al. The IL-8/CXCR1 axis is associated with cancer stem cell-like properties and correlates with clinical prognosis in human pancreatic cancer cases. Sci Rep-Uk. 2014;4:5911.

18. Highfill SL, Cui YZ, Giles AJ, et al. Disruption of CXCR2-mediated MDSC tumor trafficking enhances anti-PD1 efficacy. Sci Transl Med. 2014;6:237. doi:10.1126/scitranslmed.3007974

19. Liu YN, Chang TH, Tsai MF, et al. IL-8 confers resistance to EGFR inhibitors by inducing stem cell properties in lung cancer. Oncotarget. 2015;6(12):10415-10431. doi:10.18632/oncotarget.3389

20. Cheng HT, Yu HY, Gordon JR, Li F, Cheng JW. Effects of K11R and G31P mutations on the structure and biological activities of CXCL8: solution structure of human CXCL8 (3-72)K11R/G31P. Molecules. 2017;22:1229. doi:10.3390/molecules22071229

21. Chen Z, Chen X, Cheng HT, et al. A novel CXCL8-IP10 hybrid protein is effective in blocking pulmonary pathology in a mouse model of Klebsiella pneumoniae infection. Intl Immunopharmacol. 2018;62:40-45. doi:10.1016/j.intimp.2018.06.040

22. Kellar A, Egan C, Morris D. Preclinical murine models for lung cancer: clinical trial applications. Biomed Res Intl. 2015;2015: 621324. doi: $10.1155 / 2015 / 621324$

23. Strober W. Trypan blue exclusion test of cell viability. Curr Protoc Immunol. 2001;Appendix 3:Appendix3B.doi: 10.1002/0471142735

24. Khan MN, Wang B, Wei J, et al. CXCR $1 / 2$ antagonism with CXCL8/ Interleukin-8 analogue CXCL8 (3-72)K11R/G31Prestricts lung cancer growth by inhibiting tumor cell proliferation and suppressing angiogenesis. Oncotarget. 2015;6(25):21315-21327. doi:10.18632/ oncotarget.4066

25. Rho JK, Choi YJ, Lee JK, et al. Epithelial to mesenchymal transition derived from repeated exposure to gefitinib determines the sensitivity to EGFR inhibitors in A549, a non-small cell lung cancer cell line. Lung Cancer. 2009;63(2):219-226. doi:10.1016/j.lungcan.2008.05. 017 
26. Yu J, Ohuchida K, Mizumoto K, Fujita H, Nakata K, Tanaka M. MicroRNA miR-17-5p is overexpressed in pancreatic cancer, associated with a poor prognosis and involved in cancer cell proliferation and invasion. Cancer Biology \& Therapy. 2010;10(8):748-757. doi:10.4161/cbt.10.8.13083

27. Fernando RI, Castillo MD, Litzinger M, Hamilton DH, Palena C. IL8 signaling plays a critical role in the epithelial-mesenchymal transition of human carcinoma cells. Cancer Res. 2011;71(15):5296-5306. doi:10.1158/0008-5472.CAN-11-0156

28. Zhu YM, Webster SJ, Flower D, Woll PJ. Interleukin-8/CXCL8 is a growth factor for human lung cancer cells. British Journal of Cancer. 2004;91(11):1970-1976. doi:10.1038/sj.bjc.6602227

29. Chikara S, Lindsey K, Dhillon H, et al. Enterolactone induces G1phase cell cycle arrest in nonsmall cell lung cancer cells by downregulating cyclins and cyclin-dependent kinases. Nutr Cancer. 2017;69(4):652-662. doi:10.1080/01635581.2017.1296169

30. el-Deiry WS, Harper JW, O'Connor PM, et al. WAF1/CIP1 is induced in p53-mediated G1 arrest and apoptosis. Cancer Res. 1994;54(5):1169-1174.

31. Song XL, Zhang YJ, Wang XF, et al. Casticin induces apoptosis and G0/G1 cell cycle arrest in gallbladder cancer cells. Cancer Cell Int. 2017;17:9. doi:10.1186/s12935-016-0377-3

32. Tarasov V, Jung $\mathrm{P}$, Verdoodt $\mathrm{B}$, et al. Differential regulation of microRNAs by p53 revealed by massively parallel sequencing: miR-34a is a p53 target that induces apoptosis and G1-arrest. Cell Cycle. 2007;6(13):1586-1593. doi:10.4161/cc.6.13.4436

33. Chambers AF, Groom AC, MacDonald IC. Dissemination and growth of cancer cells in metastatic sites. Nat Rev Cancer. 2002;2(8):563572. doi: $10.1038 / \mathrm{nrc} 865$

34. Liu Q, Li A, Tian Y, et al. The CXCL8-CXCR1/2 pathways in cancer. Cytokine Growth Factor Rev. 2016;31:61-71. doi:10.1016/j.cytogfr. 2016.08.002
35. Fridman WH, Pages F, Sautes-Fridman C, Galon J. The immune contexture in human tumours impact on clinical outcome. Nat Rev Cancer. 2012;12:298-306. doi:10.1038/nrc3245

36. Luppi F, Longo AM, de Boer WI, Rabe KF, Hiemstra PS. Interleukin-8 stimulates cell proliferation in non-small cell lung cancer through epidermal growth factor receptor transactivation. Lung Cancer. 2007;56(1):25-33. doi:10.1016/j.lungcan.2006.11.014

37. Heit B, Colarusso P, Kubes P. Fundamentally different roles for LFA1, Mac-1 and alpha4-integrin in neutrophil chemotaxis. J Cell Sci. 2005;118:5205-5220. doi:10.1242/jcs.02632

38. Ibbotson GC, Doig C, Kaur J, et al. Functional alpha4-integrin: a newly identified pathway of neutrophil recruitment in critically ill septic patients. Nat Med. 2001;7:465-470. doi:10.1038/86539

39. McDonald B, McAvoy EF, Lam F, et al. Interaction of CD44 and hyaluronan is the dominant mechanism for neutrophil sequestration in inflamed liver sinusoids. $J$ Exp Med. 2008;205:915-927. doi:10.1084/jem.20071765

40. Giannoni E, Parri M, Chiarugi P. EMT and oxidative stress: a bidirectional interplay affecting tumor malignancy. Antioxid Redox Signal. 2012;16(11):1248-1263. doi:10.1089/ars.2011.4280

41. Long X, Ye Y, Zhang L, et al. IL-8, a novel messenger to cross-link inflammation and tumor EMT via autocrine and paracrine pathways (Review). Int J Oncol. 2016;48(1):5-12. doi:10.3892/ijo.2015.3234

42. Guo Y, Zang Y, Lv L, et al. IL8 promotes proliferation and inhibition of apoptosis via STAT3/AKT/NFkappaB pathway in prostate cancer. Mol Med Rep. 2017;16(6):9035-9042. doi:10.3892/mmr.2017.7747

43. Waugh DJ, Wilson C. The interleukin- 8 pathway in cancer. Clin Cancer Res. 2008;14(21):6735-6741. doi:10.1158/1078-0432.CCR$07-4843$

44. Abraham RT. Chemokine to the rescue: interleukin-8 mediates resistance to PI3K-pathway-targeted therapy in breast cancer. Cancer Cell. 2012;22(6):703-705.
OncoTargets and Therapy

\section{Publish your work in this journal}

OncoTargets and Therapy is an international, peer-reviewed, open access journal focusing on the pathological basis of all cancers, potential targets for therapy and treatment protocols employed to improve the management of cancer patients. The journal also focuses on the impact of management programs and new therapeutic

Submit your manuscript here: https://www.dovepress.com/oncotargets-and-therapy-journ agents and protocols on patient perspectives such as quality of life, adherence and satisfaction. The manuscript management system is completely online and includes a very quick and fair peer-review system, which is all easy to use. Visit http://www.dovepress.com/ testimonials.php to read real quotes from published authors. 